\title{
Pratiques enseignantes \\ Enseigner l'éducation physique en temps de pandémie
}

Auteures

Camille Godue-Couture, candidate à la maitrise en éducation, Université du Québec à Chicoutimi, Canada,

camille.godue-couture1@uqac.ca

Johanne Tremblay, enseignante en éducation physique, cégep de SaintFélicien, Canada,

johanne.tremblay@cegepstfe.ca 


\section{REVUE HYBRIDE DE L'ÉDUCATION}

\section{Résumé}

Cet article présente les changements apportés aux pratiques enseignantes de Johanne Tremblay, enseignante d'éducation physique et coordonnatrice départementale au cégep de Saint-Félicien, lors de la pandémie de la COVID-19. L'article fait état des différentes adaptations qu'elle a réalisées durant les sessions d'hiver et d'automne 2020. II met en valeur le rôle et l'apport des principaux acteurs de son milieu, de même que l'importance de la collaboration durant cette période de grand bouleversement.

Mots-clés : éducation physique; enseignement à distance; pandémie ; adaptation ; collaboration 


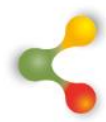

\section{REVUE HYBRIDE DE L'ÉDUCATION}

\section{Mise en contexte}

La pandémie survenue au cours de l'année 2020 a bouleversé la planète. Plusieurs secteurs ont dû rapidement s'adapter aux nouvelles mesures mises en place par nos gouvernements, le système d'éducation québécois n'en faisant pas exception. En mars 2020, le cégep de SaintFélicien, situé au Saguenay-Lac-Saint-Jean (SLSJ), a conséquemment dû interdire l'accès au bâtiment et les enseignants ont été invités à enseigner à distance. Le défi était de taille pour eux. Ils ont dû modifier leurs méthodes d'enseignement, tout en s'assurant que leurs étudiants puissent acquérir les connaissances essentielles à la passation de leurs cours. Une fois la session d'hiver terminé, les établissements scolaires se sont préparés à la rentrée des étudiants pour la session suivante. Les mesures ayant été assouplies, les cours en présentiel ont été permis avec des mesures de distanciation sociale. Or, vers la mi-session, une deuxième vague est survenue au SLSJ et a contraint le cégep de Saint-Félicien de réduire les cours en présentiel afin de minimiser les interactions sociales. Des adaptations aux pratiques enseignantes se sont donc avérées nécessaires pour pouvoir continuer de répondre aux besoins des étudiants.

Une entrevue semi-dirigée a dès lors été réalisée virtuellement auprès de madame Johanne Tremblay, enseignante et coordonnatrice du département d'éducation physique au cégep de Saint-Félicien, pour qu'elle puisse partager son expérience d'enseignement en contexte de pandémie. À la suite de cette entrevue, une ébauche de l'article a été réalisée par Camille Godue-Couture, étudiante à la maitrise en éducation, puis révisée et finalisée en étroite collaboration avec l'enseignante. Les prochaines lignes permettent de présenter cette enseignante.

Madame Tremblay enseigne l'éducation physique aux étudiants inscrits dans les programmes préuniversitaires et techniques. Elle enseigne principalement des cours de l'Ensemble ${ }^{1} 1$ (Santé et activité), de l'Ensemble 2 (Amélioration et activité) et de l'Ensemble 3 (Autonomie et activité). Elle possède une excellente connaissance du domaine d'enseignement et une maitrise du contexte collégial ce qui l'a amenée à occuper la tâche de coordonnatrice départementale. En tant que coordonnatrice, elle assure la gestion des activités départementales. En tant que représentante de son département, elle tend à être une leader pédagogique et elle assure les liens entre le département et l'administration. Elle demeure également impliquée dans son milieu de travail. Elle reconnait l'importance d'échanger avec ses collègues et elle met en avant-plan l'importance du réseau de soutien que créent ses collègues de travail au sein du cégep de Saint-Félicien.

\footnotetext{
${ }^{1}$ Dans l'ensemble des collèges, ces trois cours d'éducation physique, nommés Ensembles 1, 2 et 3 , développent chacun une compétence précise (ministère de l'Éducation et de l'Enseignement supérieur, 2016).
} 


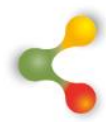

\section{REVUE HYBRIDE DE L'ÉDUCATION}

Cette praticienne d'expérience vise l'exploitation de pratiques innovantes. Depuis l'obtention de son baccalauréat en enseignement de l'éducation physique et de la santé (diplôme de premier cycle universitaire), elle n'a cessé de peaufiner sa formation. Au fil des ans, elle a suivi différents cours et formations technopédagogiques qui lui ont permis de diversifier ses pratiques et de bonifier son expertise. Elle a obtenu un diplôme d'études supérieures spécialisées (DESS) et est aujourd'hui en voie de terminer sa maitrise à l'Université de Sherbrooke. Elle s'intéresse particulièrement à la recherche et à l'innovation et s'est donné pour mission de donner envie aux étudiants de bouger, de devenir des adultes responsables aptes à prendre soin de leur santé, tant mentale que physique. En somme, il apparait que madame Tremblay valorise sa communauté professionnelle.

II va sans dire que la pandémie a nécessité des ajustements, et ce, autant à titre d'enseignante que de coordonnatrice. En effet, madame Tremblay s'est souciée d'offrir des cours de qualité, tout en étant sollicitée pour prendre des décisions rapidement en tant que coordonnatrice du département. Son sens de la créativité lui a permis d'adapter ses pratiques de plusieurs manières durant cette crise sanitaire. La prochaine section de l'article permet de confirmer le tout. En effet, elle vise à décrire les différentes adaptations que madame Tremblay a effectuées dans ses cours d'éducation physique durant la pandémie lors des sessions d'hiver et d'automne 2020.

\section{Déroulement}

\section{Hiver 2020}

Comme précisé antérieurement, les pratiques enseignantes ont été chamboulées à partir de mars 2020, lorsque le gouvernement a annoncé la fermeture des institutions collégiales. Les enseignants ont dû modifier leurs pratiques et utiliser davantage d'outils numériques pour leur permettre d'enseigner à distance. Madame Tremblay avait à ce moment plusieurs cours à sa charge.

L'un des outils numériques qui ont été grandement utilisés au cégep de Saint-Félicien est la plateforme Moodle. Moodle est une plateforme d'apprentissage en ligne. Elle favorise un apprentissage actif et dynamique. Sur celle-ci, il est possible, entre autres, d'explorer des pages web, créer des discussions de groupe, évaluer des travaux, faire des sondages et télécharger des fichiers. Cette plateforme permet de créer un environnement d'apprentissage actif qui favorise les interactions entre élèves et enseignants (Rice et William, 2006). Madame Tremblay était déjà à l'aise avec l'utilisation de l'environnement numérique Moodle puisqu'elle l'utilisait dans ses cours depuis 2012. Elle a donc eu une certaine facilité à adapter les contenus théoriques de ses cours à l'enseignement en ligne. 


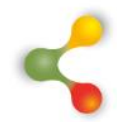

\section{REVUE HYBRIDE DE L'ÉDUCATION}

Qui plus est, ses cours théoriques se déroulaient sur la plateforme Zoom. Celle-ci permet de piloter des cours en vidéoconférence. Le partage d'écran peut également être effectué, ce qui facilite la présentation de contenus d'enseignement.

Pour madame Tremblay, le principal défi qu'elle a rencontré dans l'enseignement en ligne demeure que l'éducation physique est une discipline qui touche trois aspects de l'être humain : le physique, le mental et l'affectif. Tout comme plusieurs autres disciplines, l'éducation physique ne s'enseigne donc pas seulement sous forme magistrale. Elle nécessite de la pratique. À titre d'exemple, il est difficile d'apprendre à nager sans se pratiquer dans l'eau. Or, afin de respecter les mesures sanitaires de distanciation sociale, la présence des étudiants n'était pas permise dans les différentes installations sportives du cégep (ex. : gymnases et plateaux) à l'hiver 2020. Le volet pratique de ses cours a donc représenté tout un casse-tête. Madame Tremblay a donc dû modifier ses plans de cours et plusieurs stratégies pédagogiques innovantes ont été mises en place.

Dans un premier temps, madame Tremblay a utilisé plusieurs activités d'apprentissage en format numérique (ex. : jeux-questionnaires interactifs, examens numériques, balados et tutoriels) qu'elle avait conçues auparavant et qui permettaient de dynamiser ses cours. Selon elle, ces activités ont permis de réduire le stress et l'anxiété qui peuvent être vécus chez un enseignant qui fait face à un tel revirement de situation.

Dans un deuxième temps, pour pouvoir atteindre les objectifs de cours de chaque Ensemble, madame Tremblay a choisi de favoriser des activités de travail d'équipe en ligne. Les étudiants inscrits dans certains cours optionnels (ex. : natation, raquette ou ski de fond) étaient invités, par exemple, à filmer un partenaire afin d'améliorer un geste sportif. Chaque membre du binôme devait analyser les gestes de son partenaire en les comparant avec une vidéo retrouvée sur Internet. C'est ici qu'intervient la pédagogie par erreur.

Qui plus est, pour la portion pratique des cours, toutes les activités pratiquées ont été remplacées par la course à pied. Bien que les étudiants aient dû faire le deuil de la discipline dans laquelle ils étaient inscrits, ils ont tout de même été en mesure de développer les compétences évaluées. Pour madame Tremblay, la course à pied semblait être l'activité idéale, car elle est accessible à tous, elle se pratique de manière individuelle et à l'extérieur. Naturellement, le plan de cours a dû être adapté à cette nouvelle réalité. Madame Tremblay a donc effectué quelques accommodations, avec l'accord de la direction des études.

Dans un troisième temps, les étudiants de madame Tremblay ont eu à créer un programme d'activité physique personnel (PAPP). Pour ce faire, ils ont utilisé l'application Strava. Cette application a été très utile pour mesurer la distance de course parcourue, le temps d'exercice et la vitesse 


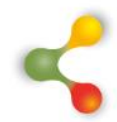

\section{REVUE HYBRIDE DE L’ÉDUCATION}

atteinte. II s'agit d'une application qui peut être téléchargée sur un téléphone intelligent et qui utilise les données GPS de celui-ci. Elle permet de suivre l'activité physique en direct et d'analyser les données après l'effort. Les étudiants se connectaient à Zoom pour leurs cours et madame Tremblay leur donnait des consignes. Ils partaient ensuite courir selon le PAPP qu'ils avaient établi. L'enseignante était en mesure de suivre chaque étudiant, en direct, sur l'application, et ce, tout au long de leur parcours. Par la suite, les étudiants devaient envoyer un rapport d'activité à leur enseignante en réalisant une capture d'écran de leur parcours détaillé.

Pour madame Tremblay, cette nouvelle façon de faire a permis à ses étudiants de bouger, d'apprendre et de rester motivés. Ces différentes adaptations n'auraient pu être réalisables sans le soutien de la direction des études et l'ouverture d'esprit des étudiants.

Une fois cette session terminée, madame Tremblay devait déjà planifier la rentrée du mois d'août. Ne sachant pas exactement à quoi s'attendre, la direction et les enseignants ont commencé à réfléchir et à adapter leurs plans de cours dans le but d'éviter d'être pris de court durant la session d'automne 2020. II fallait avoir un plan d'action dans l'éventualité où les cours devaient être proposés à distance ou selon une formule bimodale (parfois appelée hybride).

\section{Automne 2020}

Durant l'été, les mesures sanitaires ont tranquillement été assouplies au SLSJ, ce qui a permis aux établissements collégiaux d'offrir certains cours en présentiel durant la session d'automne 2020. La pandémie étant toujours d'actualité, le gouvernement a mis en place un système de paliers d'alerte guidant les mesures sanitaires selon le niveau de risques de transmission par région. Le SLSJ, qui avait été épargné durant la première vague, s'est retrouvé au palier d'alerte maximale à la fin octobre, voyant le nombre de cas dans la région augmenter à une vitesse alarmante. Ce faisant, les mesures sanitaires ont été renforcées. Les établissements collégiaux ont dû minimiser les interactions sociales et réduire les contacts à l'essentiel pour la deuxième moitié de la session.

À titre de coordonnatrice départementale, madame Tremblay voulait que les cours d'éducation physique de cette session se déroulent entièrement à l'extérieur, de façon intensive et en présentiel. Toutes les activités habituellement prévues à l'intérieur (le CrossFit, la natation, le curling, le tir à l'arc, le badminton, etc.) ont donc été bannies et remplacées par des activités extérieures telles que le vélo de montagne, la randonnée pédestre, la course, le golf, le soccer ou le canot.

Les cours étaient proposés par bloc de quatre heures en présentiel. Les activités ayant lieu à l'extérieur, il était plus facile de respecter les mesures de distanciation sociale recommandées par l'État. 


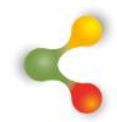

\section{REVUE HYBRIDE DE L'ÉDUCATION}

Puisque la majorité des activités nécessitaient de se déplacer à l'extérieur du campus, les frais de transport ont été plus élevés qu'à l'habitude. Les coûts supplémentaires sont principalement reliés au nombre accru d'autobus requis pour déplacer un groupe d'étudiants, puisque le respect de la distanciation sociale implique qu'un maximum de douze étudiants soit admis dans un autobus.

Puisque les cours étaient dispensés de façon intensive durant cette session, vingt-quatre heures de cours avaient déjà été effectuées sur trente heures lorsque le SLSL est passé au palier d'alerte maximale. Ne voulant pas être un vecteur de contagion, et ayant une responsabilité sociale, les enseignants du département d'éducation physique ont décidé que les six heures restantes seraient effectuées à distance. Le contenu des cours en mode virtuel était tant théorique que pratique. L'utilisation de l'application Strava et d'autres applications de mise en forme (Adidas, Nike+ Club) étaient une fois de plus pertinentes.

Pour les cours théoriques, madame Tremblay effectuait, à l'aide de la plateforme Zoom, une heure trente de théorie magistrale. Cette période était suivie d'activités pédagogiques que les étudiants devaient réaliser (ex. : carte conceptuelle, mots croisés, jeu-questionnaire, etc.), lesquelles étaient proposées avec un guide d'accompagnement. Par la suite, en sousgroupe, les étudiants comparaient leurs réponses et présentaient le résultat final de leurs discussions au reste de la classe.

En septembre, le cégep de Saint-Félicien a également été victime d'une cyberattaque. En plus d'enseigner à distance, les enseignants de ce cégep ont dû faire face à cette épreuve additionnelle. Celle-ci a bloqué toutes les plateformes électroniques de l'institution durant environ deux semaines. Les communications entre le personnel et les étudiants ont alors été très laborieuses. Pour madame Tremblay, cette étape a été la plus difficile à surmonter. Toutes les communications avec ses étudiants et le personnel ont été coupées. Les étudiants et les enseignants n'avaient également plus accès à la plateforme Moodle, ce qui représentait un défi supplémentaire pour cette enseignante puisqu'elle avait déposé tous ses contenus de cours sur cette plateforme numérique. Elle avait prévu de faire ses cours cent pour cent à l'extérieur afin d'être le plus autonome possible à la session d'automne 2020. Cette cyberattaque a été un défi, puisque la plateforme numérique qu'elle utilisait pour partager les notes de cours, les évaluations et les autres documents essentiels avec ses étudiants n'était plus accessible.

Heureusement, durant la pandémie, et encore aujourd'hui, plusieurs acteurs ont été d'une aide précieuse et ont permis aux enseignants d'être épaulés et soutenus dans ces innombrables défis afin de pouvoir accompagner les étudiants dans leurs apprentissages et leur réussite. 


\section{$\&$}

\section{REVUE HYBRIDE DE L'ÉDUCATION}

\section{Apports et prospectives}

À la suite de ce changement de réalité, une multitude d'adaptations sont survenues non seulement pour les enseignants, mais également pour les cégépiens, la direction et les membres du personnel du cégep de SaintFélicien. Madame Tremblay a compris, au fil des années, l'importance de travailler en équipe et la puissance que procure le fait d'être soutenue par une communauté de professionnels en éducation supérieure. Plusieurs acteurs de son milieu ont été importants et essentiels à la réalisation et à la réussite de son enseignement durant l'année 2020.

Selon l'enseignante, travailler seul se conjugue habituellement avec le fait d'avoir une vision en silo. En contrepartie, coopérer et travailler en équipe permet d'ouvrir ses horizons et de percevoir une même situation sous un angle différent, selon des perspectives parfois difficiles à envisager lorsqu'on est seul. Madame Tremblay favorise grandement les valeurs du socioconstructivisme qui soutiennent que l'apprentissage est actif et qu'il demeure possible d'apprendre grâce aux interactions avec nos pairs (Vienneau, 2017). Bien qu'elle incarne ces valeurs dans son enseignement, elle essaie également de les appliquer lorsqu'elle est en interaction avec ses collègues. Ayant été une athlète compétitive dans différents sports, elle est davantage dans un esprit de collaboration que de compétition lorsqu'elle est au travail. Pour elle, le réseau de soutien mis en place par l'établissement d'enseignement où elle œuvre est très important. C'est un système qui devrait servir de référence à tout enseignant. Les acteurs œuvrant au collégial ont tous un but commun et font face aux mêmes défis et réalités. Ce sont donc les meilleures personnes pour aider et comprendre la situation. II est essentiel de s'entraider afin de pouvoir avancer et atteindre les objectifs de tous, dans un même but commun : celui d'assurer la réussite des étudiants.

\section{L'apport des acteurs du milieu}

Pour faire face aux défis apportés par la pandémie, madame Tremblay souligne l'importance du réseau pédagogique et du réseau de décideurs de son établissement. Elle considère le fait d'être si bien entourée comme une force. Pour elle, la direction a été très accommodante et a permis aux enseignants de réaliser des accommodations circonstancielles. Elle a également débloqué des budgets rapidement afin de permettre aux enseignants de s'ajuster et d'avoir les outils, les ressources et les programmes nécessaires pour faciliter l'enseignement à distance.

Pour madame Tremblay, les conseillers pédagogiques du cégep de Saint-Félicien ont été extraordinaires et soucieux de la qualité de l'enseignement. Les employés du service informatique ont également été d'une grande aide et ont permis aux enseignants de se sentir soutenus et d'avoir les bons outils pour donner des cours de qualité. 


\section{$\&$}

\section{REVUE HYBRIDE DE L'ÉDUCATION}

En somme, cette enseignante d'éducation physique attribue la réussite de son enseignement en temps de pandémie à son organisation en amont de la première vague, à son sens de l'adaptation, à la collaboration des acteurs de son milieu et à la bienveillance de ceux-ci tout au long de l'année 2020.

\section{Les étudiants}

Pour madame Tremblay, il faut également reconnaitre la grande flexibilité et l'ouverture dont les étudiants ont fait preuve. Sans leur motivation et leur capacité d'adaptation, tous les efforts du personnel enseignant auraient été vains. Au cours de ces mois d'études à distance, les étudiants ont pu développer de meilleures compétences technopédagogiques. Ils ont également pu être évalués et recevoir des rétroactions multiples auxquelles ils n'étaient pas habitués, ce qui a permis de rejoindre un plus grand nombre d'apprenants. Convenons qu'ils ont développé une façon de socialiser qui leur permettait d'être en interaction les uns avec les autres, sans pour autant être physiquement présents en classe. Madame Tremblay admire ses étudiants et considère qu'ils sont demeurés résilients, compréhensifs et coopératifs. Ils ont assisté à tous les cours et sont restés motivés, peu importe la situation et les conditions météorologiques. Ils ont particulièrement aimé que leurs cours d'éducation physique soient réalisés à l'extérieur en présentiel à la session d'automne 2020, puisque c'était pour eux un des seuls moments où ils pouvaient socialiser et voir réellement leurs collègues, comme c'était le cas dans la majorité de leurs cours.

\section{Ce que l'on retient}

La coopération, la communauté, l'adaptation, la bienveillance et la planification sont des concepts clés qui ont permis à madame Tremblay de modifier ses pratiques enseignantes en temps de pandémie et de réussir à remplir les exigences de ses cours sans en compromettre la qualité. Ce qu'elle retient de cette situation, c'est d'être indulgent envers soi-même. II ne faut pas essayer de tout faire en même temps et tout seul. Selon elle, une formule d'enseignement bimodale ou hybride peut être intéressante dans les années futures. La portion théorique des cours d'éducation physique est envisageable en ligne. De plus, en cas de tempête, par exemple, il est désormais concevable, en quelques minutes, de changer la planification du cours et de l'adapter pour faire un enseignement à distance. La pandémie lui a permis de diversifier ses méthodes et d'innover de façon considérable en très peu de temps. II est normal de faire des erreurs, l'important est d'apprendre de celles-ci. Au fur et à mesure qu'elle innove, qu'elle essaie de nouvelles approches et qu'elle modifie ses pratiques enseignantes, madame Tremblay espère pouvoir inspirer d'autres enseignants. En travaillant ensemble et en apprenant des uns comme des autres, la communauté enseignante continue d'évoluer et d'innover. 


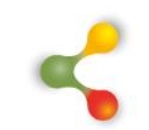

\section{REVUE HYBRIDE DE L'ÉDUCATION}

Madame Tremblay termine son entrevue en rappelant que de nombreux défis attendent encore les enseignants, mais qu'il importe de se faire confiance. C'est collectivement que tous pourront les relever. 


\section{REVUE HYBRIDE DE L'ÉDUCATION}

\section{Références}

Ministère de l'Éducation et de l'Enseignement supérieur. (2016). Composantes de la formation générale: Extraits des programmes d'études conduisant au diplôme d'études collégiales (DEC). Gouvernement du Québec.

http://www.education.gouv.qc.ca/fileadmin/site web/documents/ens eignement-

superieur/collegial/Composantes formation generale VF.pdf

Rice, W. et William, H. (2006). Moodle. Packt publishing.

Vienneau, R. (2017). Apprentissage et enseignement : théories et pratiques ( $3^{\mathrm{e}}$ édition). Gaëtan Morin éditeur. 